\title{
Backscattering from buried sediment layers: The equivalent input backscattering strength model
}

\author{
Laurent Guillon ${ }^{\text {a) }}$ \\ Laboratoire de Mécanique et d'Acoustique, UPR CNRS 7051, 31, chemin Joseph Aiguier, \\ 13402 Marseille Cedex 20, France \\ Xavier Lurton ${ }^{\text {b) }}$ \\ IFREMER, Service Acoustique et Sismique, BP 70, 29280 Plouzané, France
}

(Received 27 March 2000; revised 31 July 2000; accepted 5 October 2000)

\begin{abstract}
Signals received by low-frequency multibeam echosounders are strongly affected by sound penetration inside the upper sediment layers and by backscattering from buried layers down to depths of a few meters; this may lead to serious ambiguities and misinterpretations of experimental data. These phenomena are modeled here using a concept of equivalent input backscattering strength (EIBS), based on a combination of classical models of local backscattering strength and propagation inside fluid layered media. The local backscattering strength at a buried interface is expressed first to account for the impedance adaptation due to the overlying layers, for the angular refraction effects due to the velocity profile, and for the layered structure of the underlying medium. It is then transferred to the upper water-sediment interface, accounting for propagation inside the layered stack; the transfer coefficient is obtained from the classical theory of plane wave propagation in layered media. The volume backscattering effects are processed in the same way and account for the finite thickness of the layers. The various contributions are finally summed to give the backscattering strength, at the upper interface, that features the various effects of propagation and attenuation inside the layered structure. (C) 2001 Acoustical Society of America.
\end{abstract}

[DOI: 10.1121/1.1329622]

PACS numbers: 43.30.Gv, 43.30.Hw, 43.30.Ma, 43.30.Pc [DLB]

\section{INTRODUCTION}

Deep-sea multibeam echosounders are widespread tools in marine geology studies since they can readily provide bathymetry and sonar images of large seafloor areas. A geologist's first interpretation can be formed from these images by combining the recorded backscattering strength with the seafloor nature and assuming a one-to-one relation between these two variables. Classically, backscattering strength is modeled as the sum of one contribution from the rough surface and one from the semi-infinite volume below it (see, e.g., Ref. 1). But, at low frequencies (typically $10-15 \mathrm{kHz}$ for deep-water systems), the signal may significantly penetrate into the seafloor. Since the geological context may be very complex in the first meters of sediment, the stratification effects on the overall response should be taken into account. Simple two-component models are not able to describe the recorded backscattering strength so using them may lead to ambiguities in data interpretation. ${ }^{2}$ There is therefore a need for geoacoustic models predicting the backscattering strength of layered seafloors.

Although many studies have dealt with the influence of sediment stratification on the reflection and transmission coefficients (see, e.g., Refs. 3-5), only a few have been devoted to backscattering by layered media. One of the earliest works was Ivakin's model ${ }^{6}$ of volume backscattering for stratified sediments, which is based on a small perturbation

\footnotetext{
a) Electronic mail: guillon@1ma.cnrs-mrs.fr

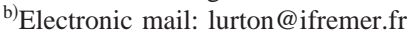

approach over a Green's function description of the acoustical field inside the sediment. Later works by Ivakin $^{7-9}$ and Tang ${ }^{10}$ proposed unified approaches of backscattering by volume and buried interfaces in layered media. They showed that if a buried interface is considered as a perturbation of the surrounding medium, the two contributions may be expressed under a single formulation.

As opposed to these "global" methods considering the problem in its generality, some authors tried to model the effects of stratification upon backscattering strength in particular configurations. Solutions were proposed for the backscattering strength of a single isotropic layer upon a basement: McDaniel ${ }^{11}$ took into account the layer roughness and Essen $^{12}$ the shear wave in the basement. They showed notable effects near the critical angle but they did not include volume backscattering strength in their models. Moreover, their exact formulations cannot be generalized to more complex geological configurations.

Various more pragmatic approaches to the problem may be found. ${ }^{2,13-15}$ In particular, Lyons et al. ${ }^{15}$ proposed a backscattering strength model for layered seafloors, in which every layer is characterized by its own individual backscattering strength (based on Jackson's model); the global backscattering strength is the sum of all the layer contributions individually modified by the layering. The layering effects, however, were not fully detailed in this attractive approach since those authors focused on volume backscattering modeling.

The principle of our equivalent input backscattering strength (EIBS) model ${ }^{16-19}$ is basically an extension of that 
of Lyons et al. ${ }^{15}$ Starting from the same intuitive description of local contributions from buried layers "seen" through the filter of an overlying sediment stack, we account for stratification effects in a more rigorous way and under a more easily generalizable formalism, (1) by carefully accounting for the local modifications of backscattering strength due to burying and (2) by using a classical model of plane wave propagation inside a fluid layered medium. Finally, the ultimate ambition of our EIBS model is to provide results in good agreement (despite their narrower generality) with those obtained from more global approaches such as Ivakin's. ${ }^{9}$

\section{THE CONCEPT OF EQUIVALENT INPUT BACKSCATTERING STRENGTH}

\section{A. Geoacoustic model}

In the following, the seafloor is described by a two-part geoacoustic model (Fig. 1):

A fluid dissipative sedimentary layer of thickness $h$, split into $n$ elementary layers. Each layer $l$ is characterized by its sound speed $c_{l}$, density $\rho_{l}$, attenuation coefficient $\alpha_{l}$, its thickness $d_{l}$, and its own individual backscattering cross section (BCS) $\sigma_{l}\left(\theta_{0}\right)$, considered at its upper boundary.

(ii) A semi-infinite fluid dissipative basement, with parameters $c_{n+1}, \rho_{n+1}$, and $\alpha_{n+1}$, and its BSC $\sigma_{n+1}\left(\theta_{0}\right)$.

Note that the concept of "basement" here relates only to the acoustical penetration, meaning that there is no significant return of energy from the medium below interface $n$ +1 . It is not related to a particular geological structure.

In the following, to define its individual BCS, each layer is first considered as having its upper boundary overlaid with water. We found this convention convenient for numerous practical configurations in which one has to compare backscattering strength levels from the same boundary either outcropping or buried under a stratified sediment layer. An initial local BCS is defined under this assumption, classically split into two components (surface roughness and volume inhomogeneities) and is then modeled using a local backscattering strength model such as Jackson's. ${ }^{1}$

\section{B. The equivalent input backscattering strength}

Calculating the total BCS features two steps. First, the local individual BCS $\sigma_{l}\left(\theta_{0}\right)$ are defined taking into account the effects due to layering: changes in impedance contrasts, influence of underlying layers, volume limitation, and refraction. These various effects are detailed in Sec. III. The modified individual BCS for each layer $l$ is written $\widetilde{\sigma}_{l}\left(\theta_{l-1}\right)$; this notation includes the angle changes due to refraction. The second step of the process is to calculate the transfer coefficients accounting for sound propagation inside the stack. The contribution of each layer $l$ is weighted by a coefficient $C_{p l}\left(\theta_{0}\right)$ determined by the acoustic field inside the layered structure. Under a small perturbation hypothesis, these coefficients can be obtained by a classical plane wave approach of sound transmission in layered media (see, e.g., Ref. 20).

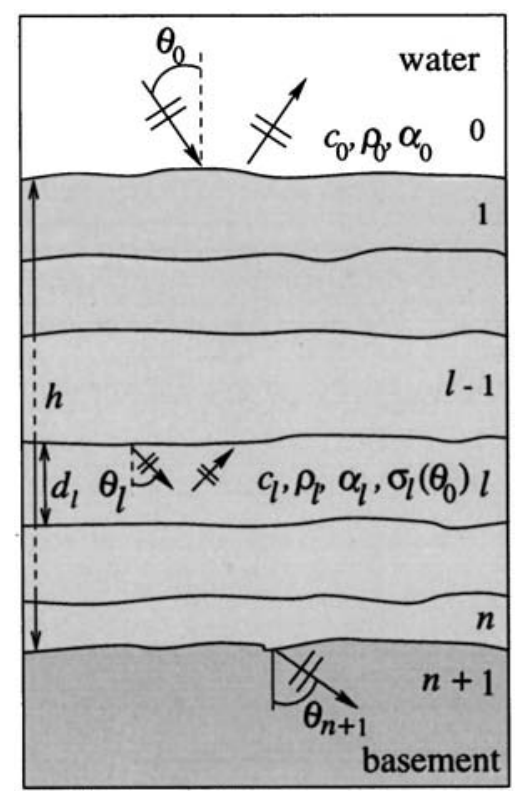

FIG. 1. Geoacoustic model.

These two steps finally lead to the "equivalent input backscattering strength" at the upper water/sediment interface. ${ }^{18}$ This term was chosen by analogy with the concept of "equivalent input impedance" in the theories of electric circuits or sound propagation in layered media. ${ }^{20}$ Thereafter, under the hypothesis of single scattering, the various contributions are summed to provide the total BCS of the geoacoustic configuration:

$$
\sigma_{t}\left(\theta_{0}\right)=\sum_{l=1}^{n+1} C_{p l}\left(\theta_{0}\right) \widetilde{\sigma}_{l}\left(\theta_{l-1}\right)
$$

The global backscattering strength for the stack is finally $10 \log \left[\sigma_{t}\left(\theta_{0}\right)\right]$.

Two hypotheses are necessary for establishing this expression of the total BCS. First, a small perturbation approach is used here: the scattered wave has second-order magnitude relative to the incident field, so one can deal with plane wave propagation inside the stack. This approximation is usually admitted in sediment backscattering models. ${ }^{1,6,21,22}$ Second, a single-scattering approach, related to the former hypothesis, is needed. The multi-scattered field is considered as negligible relative to the single-scattered echo (whose backwards propagation accounts for the complete description of phenomena associated with layered media). Consequently, one can make a single summation of the various contributions. This second hypothesis is valid if each layer's backscattering strength is low enough. This is true outside the "specular" regime, typically for incidence angles greater than 20 degrees; on the other hand, at low incidences, backscattering strength may be large enough to allow multiple scattering. Consequently, for angles close to the vertical incidence, the model must be considered as a first approximation of the problem and further calculations should be done to extend it exactly to the whole angular range. 
TABLE I. Number of parameters used for the equivalent input backscattering strength model of an $n$-layer sediment stack.

\begin{tabular}{cl}
\hline \hline \multicolumn{1}{c}{ Parameters } & No. of parameters \\
\hline Geoacoustic configuration & $n+1$ \\
$c_{l}:$ sound speed & $n+1$ \\
$\rho_{l}:$ density & $n+1$ \\
$\alpha_{l}:$ attenuation & $n$ \\
$d_{l}:$ thickness & \\
Backscattering parameters & $2 \times(n+1)$ \\
Surface & $n+1$ \\
Volume & $7 n+6$ \\
Total & \\
\hline \hline
\end{tabular}

\section{Number of parameters and discussion}

For every layer, the composite roughness model ${ }^{1}$ served to determine the individual interface BCS. We used the two classical parameters $\gamma$ and $\beta_{J}$ defining the roughness spectrum as $W_{s}(\mathbf{K})=\beta_{J} K^{-\gamma}$, and a single parameter $\sigma_{v}$ for the volume contribution.

Table I features the parameters involved in this model for the geoacoustic configuration presented in Fig. 1, showing that $7 n+6$ input parameters are needed for an $n$-layer configuration. For example, a simple two-layer model (a basement below two sediment layers) requires 20 input parameters. The effective number of independent parameters can be reduced by taking into account relations between some of them (see, e.g., Ref. 23), but the array size will nevertheless remain large.

\section{LOCAL DESCRIPTION OF THE INDIVIDUAL BCS}

Our objective was to model the modifications of the backscattering strength due to layering. To do so, we used the model of Jackson ${ }^{1}$ for the local backscattering strength of a given layer but other models could have been used. In the following, the changes induced by layering on the individual layer BCS are illustrated by numerical applications computed on a geoacoustic model with two layers (mud and medium sand) overlying a coarse sand basement. The layering effects are visualized on the figures representing the backscattering strength of the medium-sand layer. The configuration parameters are given in Table II. The acoustical parameters $(c, \rho$, and $\alpha)$ are from data compilations by Hamilton. ${ }^{23}$ The values for $\beta_{J}$ and $\gamma$ are from Mourad and

TABLE II. Input parameters of the geoacoustic model used for illustrating the numerical applications in Secs. III and IV.

\begin{tabular}{lcccc}
\hline \hline \multicolumn{1}{c}{ Layers } & Water & Mud & Medium sand & Coarse sand \\
\hline$c\left(\mathrm{~m} \cdot \mathrm{s}^{-1}\right)$ & 1500 & 1550 & 1650 & 1840 \\
$\rho$ & 1 & 1.3 & 1.43 & 2.2 \\
$\alpha\left(\mathrm{dB} / \lambda^{\mathrm{a}}\right)$ & 0 & 0.2 & 0.87 & 0.9 \\
$\gamma$ & $\ldots$ & 3.25 & 3.25 & 3.25 \\
$\beta_{J}\left(\mathrm{~cm}^{4}\right)$ & $\ldots$ & $4 \times 10^{-4}$ & $30 \times 10^{-4}$ & $60 \times 10^{-4}$ \\
$\sigma_{v}\left(\mathrm{~dB} \cdot \mathrm{m}^{-3}\right)$ & $\ldots$ & -43 & -35 & -30 \\
$d(\mathrm{~cm})$ & $\ldots$ & 20 & 10 & $\cdots$ \\
\hline \hline
\end{tabular}

${ }^{\mathrm{a}} \lambda$ is the wavelength.

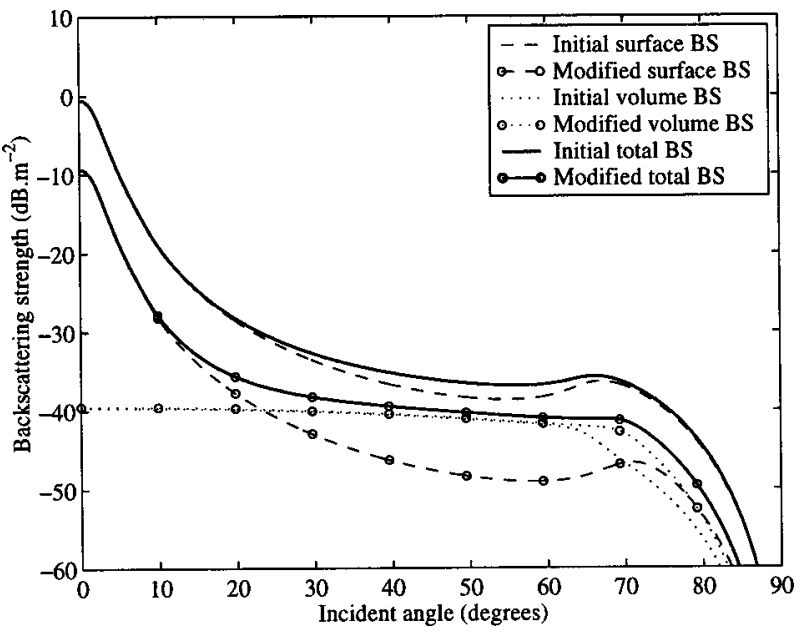

FIG. 2. Effect of impedance contrast changes for the medium sand backscattering strength (BS). The model used is Jackson's (Ref. 1) with input parameters given in Table II. The curves labeled "Initial BS", are computed for the medium-sand layer covered with water whereas the curves labeled "Modified BS", are computed for this layer covered with mud.

Jackson. ${ }^{24}$ The volume backscattering strength coefficient was based on typical values obtained on real data. The signal frequency is $13 \mathrm{kHz}$.

\section{A. Local backscattering strength definition}

As said above, for each layer $l(l>1)$, the individual initial BCS is first defined as having its interface overlaid with water. Now, when this layer is inside the sediment stack, some changes obviously occur. First, the presence of the upper sediment layer $l-1$ decreases the impedance contrast associated with the backscattering phenomenon, and this decrease modifies the local reflection and transmission coefficients. Because these coefficients are used in the local backscattering models, the individual BCS will be modified accordingly, providing a new local BCS.

As an example, Fig. 2 shows the effect of this impedance contrast change on the backscattering strength of the medium-sand layer and its clear dependence on incident angle.

At steep incidence, the backscattering process is dominated by the roughness contribution; it is modeled, in Jackson's approach, ${ }^{1}$ using Kirchhoff's approximation, as follows:

$\sigma_{k}\left(\theta_{0}\right)=\frac{R^{2}(0)}{8 \pi \cos ^{2} \theta_{0} \sin ^{2} \theta_{0}} \int_{0}^{\infty} \exp \left(-q u^{\gamma-2}\right) J_{0}(u) u d u$,

where $q$ is a function of $\theta_{0}, k, \gamma$, and $\beta_{J}$, and $R\left(\theta_{0}\right)$ is the amplitude reflection coefficient for the water-sediment boundary for incident angle $\theta_{0}$.

This BCS expression is proportional to the squared pressure reflection coefficient $R(0)$ upon the interface; hence a change from the water-sand boundary $[R(0)=0.222]$ to the mud-sand boundary $[R(0)=0.079]$ leads to a decrease of $20 \log (0.079 / 0.222)=-9.0 \mathrm{~dB}$, which is clear in Fig. 2. This is an impedance matching effect: the decrease in impedance contrast lowers the scattered energy. 
At oblique incidence, the combination of surface and volume contributions makes the interpretation more difficult. In Ref. 24, the roughness scattering at large incidence is expressed using the composite roughness model ${ }^{1}$ with the local BCS written as follows:

$$
\sigma_{c r}\left(\theta_{0}\right)=4 k^{4} \cos ^{4} \theta_{0}\left|Y\left(\theta_{0}\right)\right|^{2} W_{s}\left(2 k_{0} \sin \theta_{0}\right),
$$

where

$$
Y\left(\theta_{0}\right)=\frac{(\rho-1)^{2} \sin ^{2} \theta_{0}+\rho^{2}-\kappa^{2}}{\left[\rho \cos \theta_{0}+P\left(\theta_{0}\right)\right]^{2}} .
$$

In Eq. (4), $\rho=\rho_{1} / \rho_{0}, \kappa=k_{1} / k_{0}$, and $P\left(\theta_{0}\right)=k_{z 1} / k_{0}$, where $k_{z 1}=\sqrt{k_{1}^{2}-k_{0}^{2} \sin ^{2}\left(\theta_{0}\right)} ; \rho_{0}$ and $k_{0}$ are the density and wave number in water, these are respectively $\rho_{1}$ and $k_{1}$ in sediment. Figure 2 shows that roughness scattering at oblique incidence is also affected by the impedance matching.

For volume backscattering, we used the expression from the model of Jackson and Briggs: ${ }^{25}$

$$
\sigma_{s v}\left(\theta_{0}\right)=\frac{\left|1-R^{2}\right|^{2} \cos ^{2} \theta_{0}}{4 k_{0} \mathcal{I}\left[P\left(\theta_{0}\right)\right]\left|P\left(\theta_{0}\right)\right|^{2}} \sigma_{v} .
$$

The effect of an impedance contrast change on volume backscattering strength is weak because $\left|1-R^{2}\right|^{2}$, which features the two-way transmission losses through the watersediment interface, varies little with the impedance contrast. More important is the angular dependence effect: lowering the impedance contrast increases the critical angle, as shown in Fig. 2 and described in Sec. III D.

To summarize, the balance between the local BCS components is modified: the surface BCS is lowered (impedance matching effect) whereas the volume BCS is almost unchanged. Consequently, burying the various layers may strongly affect their BCS. Results then depend on the respective proportion of surface and volume backscattering strength in the initial BCS.

\section{B. Influence of underlying layers}

The change in impedance contrast affects the backscattering strength through the local reflection and transmission coefficients. But the underlying stratified structure also modifies these coefficients in a global way which also changes the backscattering strength. This was shown by Moe and Jackson ${ }^{26}$ for a stratified structure underlying a rough surface. In the following we used their expression for the resulting BCS:

$$
\begin{aligned}
\sigma_{s}\left(\theta_{0}\right)= & \left(\frac{k_{0}}{4}\right)^{4}\left|1+R_{L}\right|^{4} \mid 1-\frac{\kappa^{2}}{\rho}+\left(1-\frac{1}{\rho}\right) \\
& \times\left.\left[\sin ^{2} \theta_{0}+\rho \cos ^{2} \theta_{0}\left(\frac{1-R_{L}}{1+R_{L}}\right)^{2}\right]\right|^{2} W_{s}\left(2 k \sin \theta_{0}\right),
\end{aligned}
$$

where $R_{L}$ is now the amplitude reflection coefficient for the layered media (see, e.g., Ref. 20). Figure 3 presents this calculation for the medium-sand layer; to make interpretation easier the impedance contrast changes described earlier were omitted. The strong oscillations between 50 degrees and 60

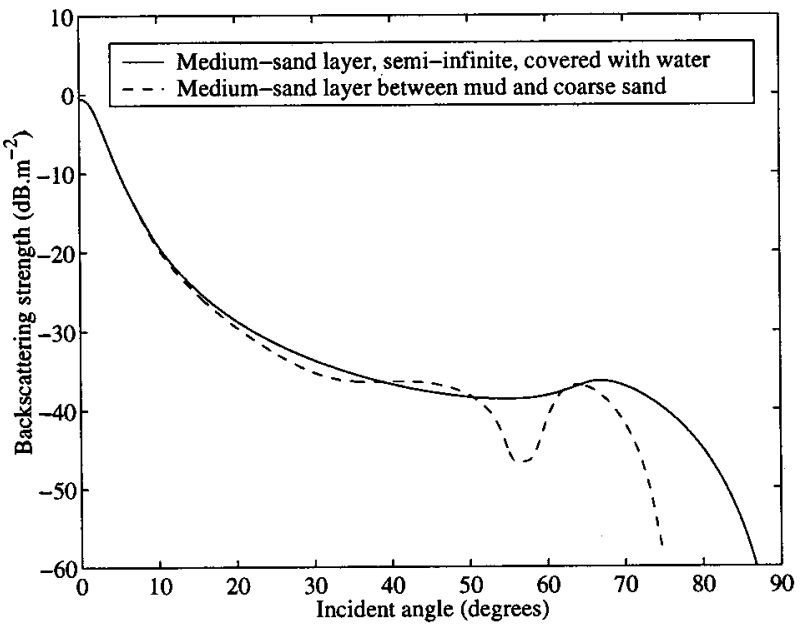

FIG. 3. Roughness backscattering strength of the medium sand layer with parameters provided in Table II. The solid curve is the initial roughness backscattering strength, and the dashed curve corresponds to this layer inside the sediment stack, using Eq. (6).

degrees are caused by the reflection on the medium-sand layer.

\section{Volume limitation}

The volume contribution to the backscattering process is classically ${ }^{25}$ modeled with a single parameter $\sigma_{v}$, which is the local BCS associated with a unit volume. This BCS is not "seen" directly, but rather through the interface. Equation (5) given above is based on the hypothesis that the scattering medium is semi-infinite. However, for a given sediment layer, this is not true any more. To take into account this volume limitation, expression (5) now becomes

$$
\left.\sigma_{s v}=\frac{\left|1-R^{2}\right|^{2} \cos ^{2}\left(\theta_{0}\right)}{4 k_{1} \mathcal{I}\left[P\left(\theta_{0}\right)\right]\left|P\left(\theta_{0}\right)\right|^{2}}\left[1-e^{-4 \mathcal{I}\left(k_{z l}\right) d}\right]\right] \sigma_{v},
$$

where $\mathcal{I}\left(k_{z l}\right)$ is the imaginary part of the vertical component of the wave vector inside the sediment. The bracketed corrective term in Eq. (7) features the attenuation effect along the layer thickness $d_{l}$.

Figure 4 presents this effect of thickness limitation on the volume backscattering strength of the medium-sand layer. Backscattering strength is lowered, with a maximum influence at steep angles. Beyond the critical angle, this effect disappears because penetration becomes negligible; the energy is conducted only by an evanescent lateral wave. ${ }^{20}$ The layer thickness is then of little importance.

\section{Refraction and attenuation}

Sound propagation inside the sediment layers implies two consequences for local individual BCS. First, the incident wave is refracted. At each interface, the SnellDescartes relation gives

$$
k_{l-1} \sin \theta_{l-1}=k_{l} \sin \theta_{l}=k_{0} \sin \theta_{0},
$$

so layer $l$ is now "seen" at incident angle $\theta_{l-1}$ instead of at the in-water original angle $\theta_{0}$. This modifies the backscattering strength angular dependence by a kind of "anamorphosis." Second, the sediment layers are dissipative. Their at- 


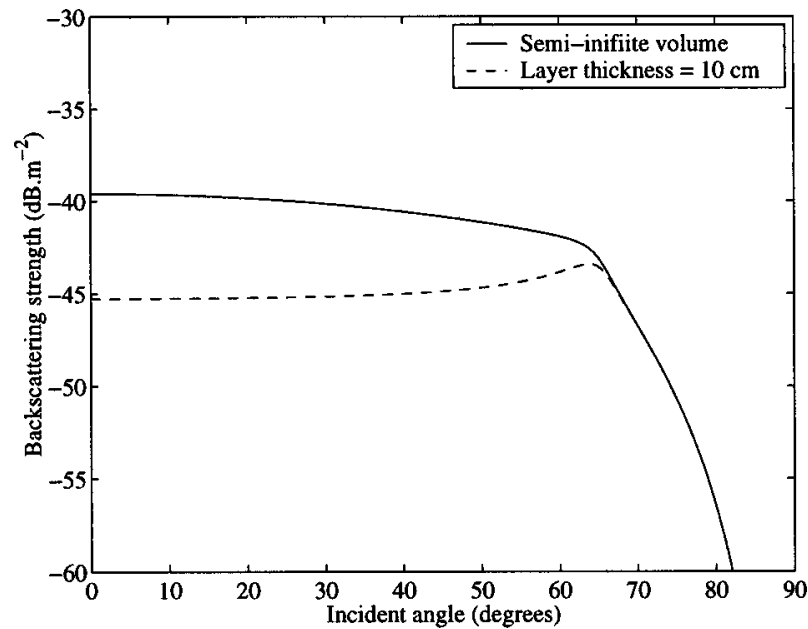

FIG. 4. Volume limitation effect on the medium-sand layer backscattering strength. Solid curve is computed with Eq. (5) whereas the dashed one corresponds to Eq. (7).

tenuation lowers the backscattered level, as seen from Eq. (7). This effect is all the stronger as the incident angle is large (the acoustical path is longer) and the layer is deep.

\section{E. Local backscattering strength synthesis}

Figure 5 summarizes the various effects described earlier that affect the individual backscattering strength of the buried medium-sand layer. On most of the angular range there is a global lowering, between 5 and $10 \mathrm{~dB}$, relative to the water-overlaid case. Moreover, the cutoff angle effect is steeper than previously. This change in critical angle is due to refraction as described in Sec. II D. The underlying stratification influence [Eq. (6)] is weak in this case because the dominant effect in this angular range is volume scattering rather than interface roughness.

\section{COMPUTATION OF THE TRANSFER COEFFICIENTS}

Now that every layer's local BCS is defined and specified, it has to be transferred upwards to the water-sediment

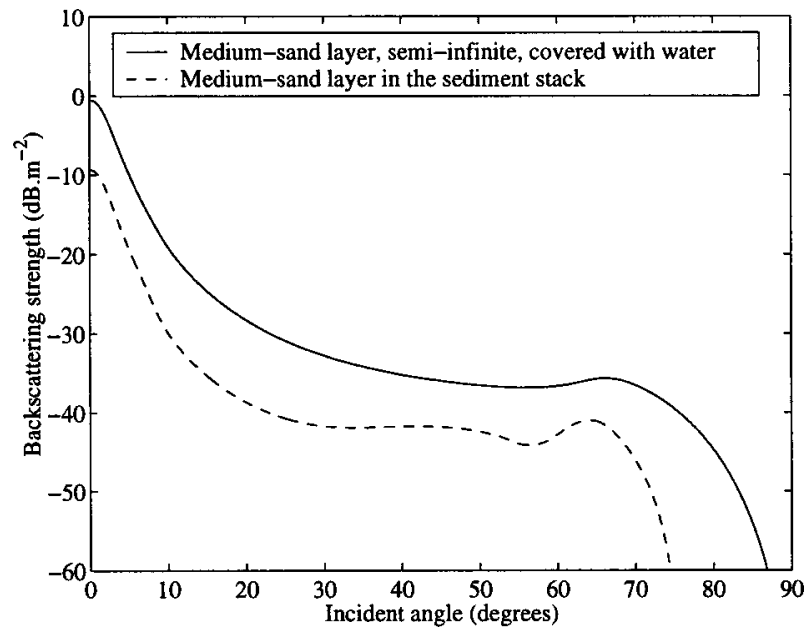

FIG. 5. Medium-sand backscattering strength as a semi-infinite medium covered with water (solid curve) and as a layer inside the sedimental stack (dashed curve). The parameters used are given in Table II.

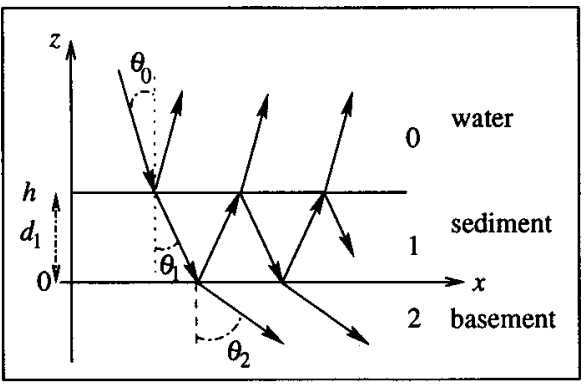

FIG. 6. Geometry for the incident path.

interface in a way correctly accounting for the propagation inside the sedimentary medium. In the following, the transfer coefficient $C_{p l}\left(\theta_{0}\right)$ quantifies, for each buried layer $l$, the total energy incident at angle $\theta_{0}$ transmitted inside the sediment down to layer $l$ and backscattered to the sonar in the same direction $\theta_{0}$. The computation of these transfer coefficients is first presented for the simple case of a single sediment layer overlying a basement and then for the general multilayered case. In the following $R_{i, j}$, or $T_{i, j}$, denotes the reflection, or transmission, coefficient from medium $i$ to medium $j$. The complex wave number $k_{l}=\omega / c_{l}+i \alpha_{l} / 8.686 \lambda$ (with $\alpha_{l}$ in $\mathrm{dB} /$ wavelength) accounts for sound attenuation in layer $l$. The vertical, or horizontal, projection of the wave vector $\mathbf{k}_{l}$ is $k_{z_{l}}$, or $k_{x_{l}}$.

\section{A. A single-sediment layer}

We develop here the basement transfer coefficient: $C_{p 2}\left(\theta_{0}\right)$. The calculation is split into two parts: we expressed first the energy incident from the water to the basement and then the energy transmitted back to the receiver.

\section{Incident path}

Figure 6 depicts the first part of this approach. Under the small perturbation hypothesis, only plane waves are considered inside the sediment layers.

The acoustical pressure field in the three media may be written as follows:

$$
\begin{aligned}
& p_{0}=\left[e^{-i k_{z_{0}}(z-h)}+R_{a} e^{i k_{z_{0}}(z-h)}\right] e^{i k_{x} x} e^{-i \omega t}, \\
& p_{1}=\left[A_{1} e^{-i k_{z_{1}} z}+B_{1} e^{i k_{z_{1}} z}\right] e^{i k_{x} x} e^{-i \omega t}, \\
& p_{2}=T_{a} e^{-i k_{z_{2}} z} e^{i k_{x} x} e^{-i \omega t}
\end{aligned}
$$

where $R_{a}$ is the reflection coefficient at the water-sediment interface for the whole sediment stack. Here $T_{a}$ is the global transmission coefficient from water to basement $\left(T_{0,2}\right)$ : ${ }^{20}$

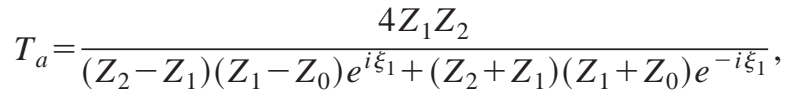

where $Z_{l}=\rho_{l} \omega / k_{z_{l}}$ is the plane wave impedance in layer $l$ and $\xi_{l}=d_{l} k_{z_{l}}$. For notation convenience, the time dependence $e^{-i \omega t}$ and the horizontal components $e^{i k_{x} x}$ are suppressed in the following, and the incident acoustical pressure is normalized to 1 . The acoustical continuity conditions at the basement boundary are used to express coefficient $A_{1}$, which is the relative amplitude of the incident wave on the considered scattering boundary: 


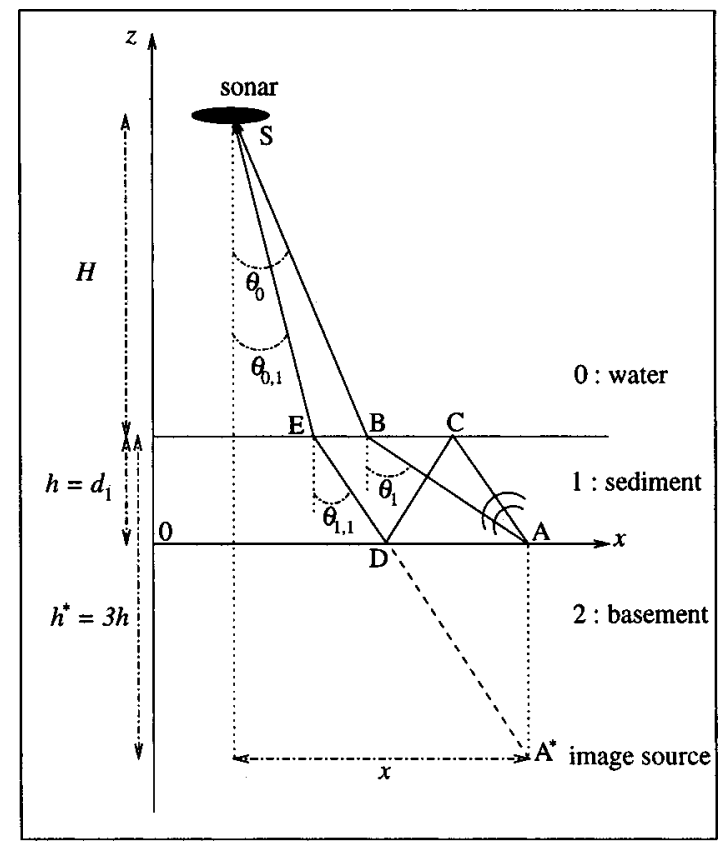

FIG. 7. Backscattered spherical wave: direct and first multiple path.

$A_{1}=T_{a} \frac{Z_{2}+Z_{1}}{2 Z_{1}}=\frac{T_{a}}{T_{1,2}}$.

The incident acoustical intensity on the sediment $(z$ $=h)$ is

$$
I_{i 0}=\frac{\cos \theta_{0}}{2 \rho_{0} c_{0}} .
$$

On the basement, the incident intensity is

$$
I_{i 1}=\frac{\left|A_{1}\right|^{2} \mathfrak{R}\left(k_{z_{1}}\right)}{2 \omega \rho_{1}},
$$

with $\mathfrak{R}(k)$ denoting the real part of the complex number $k$.

\section{Backscattered wave}

The incident acoustical wave is scattered in all directions by the basement roughness; the angular dependence is given by a scattering function. ${ }^{27}$ The energy scattered upwards through the sediment layer can therefore follow various paths back to the receiver, with different angles corresponding to image-sources due to successive reflections inside the layer; the $m$ th-order image source is at depth $z_{m}$ $=-2 m h$. Figure 7 depicts the direct path (ABS) from a scatterer at point A to the sonar and, for instance, the first multiple path (ACDES) from the first-order image source $A^{*}$ at depth $z=-2 h$.

The field emitted from $\mathrm{A}$ and transmitted to S may therefore be written as the summation of these image-source contributions:

$p_{s}\left(\theta_{0}\right)=\sum_{m=0}^{\infty} \frac{T_{1,0}\left(\theta_{1, m}\right) R_{1,0}^{m}\left(\theta_{1, m}\right) R_{1,2}^{m}\left(\theta_{1, m}\right)}{\mathcal{R}_{m}} e^{i\left[k_{0} r_{0, m}+k_{1} r_{1, m}\right]}$.

For the $m$ th image source, $r_{0, m}$ and $r_{1, m}$ are the path lengths in media 0 and $1, \theta_{1, m}$ is the incident angle inside medium 1 , and $1 / \mathcal{R}_{m}$ is the spherical loss from the image

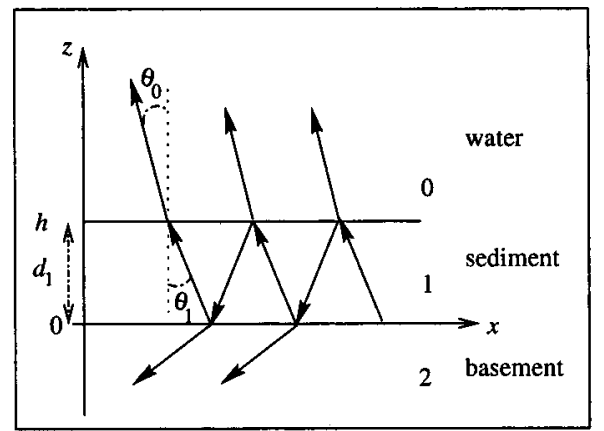

FIG. 8. Geometry for the backscattered wave.

source to the receiver. The difference in phase terms $\varphi_{m}$ for source $(m)$ in Eq. (14) and $\varphi_{0}$ for the direct path ABS is obtained by

$$
\begin{aligned}
\varphi_{m}-\varphi_{0} & =k_{0}\left(r_{0, m}-r_{0}\right)+k_{1}\left(r_{1, m}-r_{1}\right) \\
& =k_{0} H\left(\frac{1}{\cos \theta_{0, m}}-\frac{1}{\cos \theta_{0}}\right)+k_{1} h\left(\frac{2 m+1}{\cos \theta_{1, m}}-\frac{1}{\cos \theta_{1}}\right),
\end{aligned}
$$

where $\theta_{0}$ and $\theta_{1}$ are angles in media 0 and 1 for the direct path.

Since $h \ll H$ (in a typical deep-water echosounding configuration, $h$ is a few meters whereas $H$ is 1 to $5 \mathrm{~km}$ ), angle $\theta_{0, m}$ may be considered as a small perturbation of $\theta_{0}$ according to variations of $2 \mathrm{mh}$. Developing Eq. (15) to the first order gives (see details in the Appendix):

$$
\varphi_{m}-\varphi_{0}=2 m k_{1} h \cos \theta_{1, m} .
$$

The condition $h \ll H$ allows the spherical loss to be approximated as $1 / \mathcal{R}_{0} \approx 1 / \mathcal{R}_{1} \approx 1 / \mathcal{R}_{2} \approx \cdots$, meaning that the extra range and the refraction effect raised by the layer are negligible in the geometrical divergence loss. Also, the reflection and transmission coefficients may be approximated as $T_{1,0}\left(\theta_{1}\right) \approx T_{1,0}\left(\theta_{1,1}\right) \approx T_{1,0}\left(\theta_{1,2}\right) \approx \cdots \quad$ and $R_{1,0}\left(\theta_{1}\right)$ $\approx R_{1,0}\left(\theta_{1,1}\right) \approx R_{1,0}\left(\theta_{1,2}\right) \approx \cdots$ because of the very slight variation of the incident angle. This leads to the following expression for Eq. (14):

$p_{s}\left(\theta_{0}\right)=\frac{T_{1,0}\left(\theta_{1}\right)}{\mathcal{R}_{0}} e^{i \varphi_{0}} \sum_{m=0}^{\infty} R_{1,0}^{m}\left(\theta_{1}\right) R_{1,2}^{m}\left(\theta_{1}\right) e^{2 i k_{1} h m \cos \theta_{1}}$,

which features the classical expression ${ }^{20}$ for a transmission coefficient in a layered medium. Hence, the backscattered wave will be considered as one plane wave emitted upwards in direction $\theta_{1}$ (Fig. 8).

The water-transmitted field is now determined from

$$
\begin{aligned}
& p_{0}=D e^{i k_{z_{0}}(z-h)}, \\
& p_{1}=F e^{i k_{z_{1}} z}+C e^{-i k_{z_{1}} z} .
\end{aligned}
$$

For computational convenience, it is now supposed that the system depicted in Fig. 8 is generated by a virtual plane wave coming from the basement with amplitude $L$. Thus, $F$ $=T_{2,1} L$ and $D=T_{r} L$, where $T_{r}$ is the transmission coefficient from basement to water $\left(T_{2,0}\right)$ that can be obtained with 


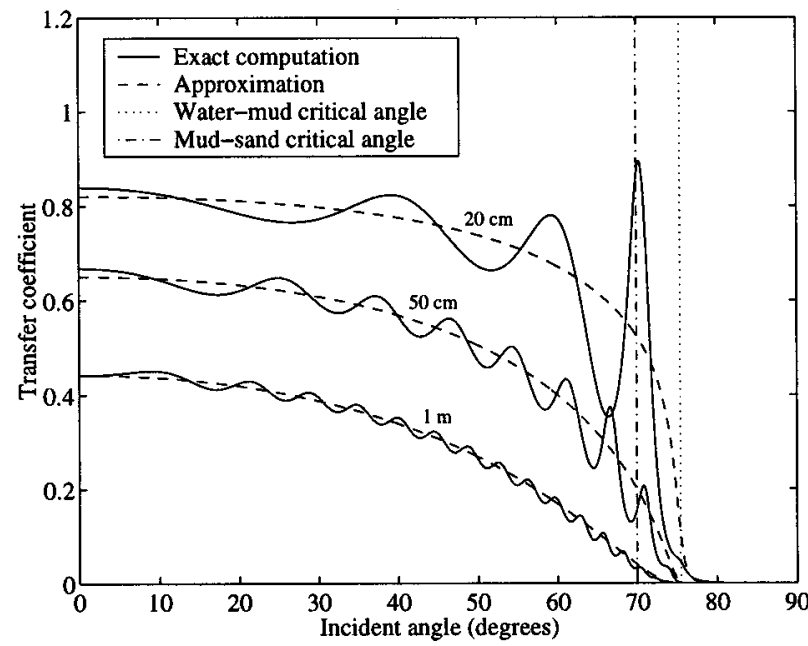

FIG. 9. Transfer coefficients computed from sand layer to water, for different mud thicknesses according to exact formulation [Eq. (24)] (solid curves) or to approximate expression [Eq. (25)] (dashed curves).

Eq. (10) by inverting indices 0 and 2. The following relation between $D$ and $F$ is obtained:

$$
D=F \frac{T_{r}}{T_{2,1}} .
$$

Inside the sediment layer, the basement-backscattered field is linked with the incident field by the relation

$$
I_{d}=\widetilde{\sigma}_{2}\left(\theta_{1}\right) I_{i 1},
$$

with the intensity $I_{d}=|F|^{2} \Re\left(k_{z_{1}}\right) / 2 \omega \rho_{1}$. Using Eq. (13), one obtains

$$
|F|^{2}=\left|A_{1}\right|^{2} \widetilde{\sigma}_{2}\left(\theta_{1}\right) .
$$

The outgoing intensity is written

$$
I_{s}=\frac{|D|^{2} \cos \theta_{0}}{2 \rho_{0} c_{0}}=|D|^{2} I_{i 0} \text {. }
$$

\section{Synthesis}

Using relations (21), (22), and (19), one can write for the intensity backscattered and transmitted back to the water

$$
I_{s}=\widetilde{\sigma}_{2}\left(\theta_{1}\right)|A|^{2}\left|\frac{T_{r}}{T_{2,1}}\right|^{2} I_{i} \text {. }
$$

Referring to Eqs. (1) and (11), the transfer coefficient for the basement underlying a single-sediment layer is finally

$$
C_{p 2}=\left|\frac{T_{a}}{T_{1,2}}\right|^{2}\left|\frac{T_{r}}{T_{2,1}}\right|^{2} \text {. }
$$

If one neglects the multiple reflected paths inside the sediment stack, this transfer coefficient becomes

$$
\breve{C}_{p 2}=\left|T_{1,0}\right|^{2}\left|T_{0,1}\right|^{2} e^{-4 h \mathcal{J}\left(k_{1}\right) / \cos \theta_{1}} .
$$

Figure 9 presents the computation results of Eqs. (24) and (25) for a medium-sand layer covered with various thicknesses of mud, for parameters given in Table II.

The transfer coefficients are maximum at steep incidences and decrease until the cutoff imposed by the critical angles of the various interfaces. These coefficients tend to zero beyond the highest critical angle. Oscillations associated with the resonant character of sound propagation inside layers are superimposed over this global trend. The density of these oscillations depends on the number of resonant angles at a given frequency, whereas their levels depend on the relative levels of interfering multipaths and hence upon attenuation across the dissipative layers. These oscillations therefore get closer and smoother when the layer thickness increases. In the case shown in Fig. 9, the effect of multipath interference is clearly negligible for the 1-m thickness, and the simple expression (25) is then a good approximation. On the other hand, for thinner mud layers, Eq. (25) is less acceptable, especially close to the critical angle cutoff where the oscillation effect becomes dominant. These oscillatory interference effects are obviously encountered in the resulting transferred backscattering strengths. Experimental evidence of such oscillations may be found in the literature (see, e.g., Ref. 13).

\section{B. General case}

In the case of a multilayered seafloor as sketched in Fig. 1 , the transfer coefficients are obtained by generalizing the single-layer case. Thus, for layer $l$ the transfer coefficient can be written as

$$
C_{p l}=\left|A_{l-1}\right|^{2}\left|\frac{T_{l, 0}}{T_{l, l-1}}\right|^{2},
$$

where $A_{l-1}$ is the incident amplitude on layer $l$, obtained from the following recursive relations:

$$
\begin{aligned}
& A_{l-1}=\frac{A_{l} e^{-i \xi_{l}}}{T_{l-1, l}}+B_{l} e^{i \xi_{l}} \frac{Z_{l}-Z_{l-1}}{2 Z_{l}}, \\
& B_{l}=A_{l-1} \frac{Z_{l-1}-Z_{l}}{2 Z_{l-1}}+B_{l-1} \frac{e^{i \xi_{l-1}}}{T_{l, l-1}},
\end{aligned}
$$

with

$$
A_{n}=\frac{T_{a}}{T_{n, n+1}}, \quad B_{n}=T_{a} \frac{Z_{n+1}-Z_{n}}{2 Z_{n+1}} .
$$

Now $T_{a}$ is the transmission coefficient from water to basement $\left(T_{0, n+1}\right)$ for the whole sediment stack: ${ }^{20}$

$$
T_{a}=\prod_{j=n+1}^{1} \frac{Z_{i n}^{(j)}+Z_{j}}{Z_{i n}^{(j)}+Z_{j-1}} e^{i \xi_{j}},
$$

with

$$
Z_{i n}^{(j)}=Z_{j} \frac{Z_{i n}^{(j+1)}-i Z_{j} \tan \xi_{j}}{Z_{j}-i Z_{i n}^{(j+1)} \tan \xi_{j}} ;
$$

and $Z_{i n}^{(n+1)}=Z_{n+1} \cdot Z_{i n}^{(2)}$ is the input impedance of the system.

Figure 10 presents the sand-to-water and basement-towater transfer coefficients. The former is limited by the water-mud critical angle, and the angle range of the latter is narrower because of the steeper angle associated with the water-sand contrast. The differences in global levels are easily accounted for by the respective sediment thicknesses considered. 


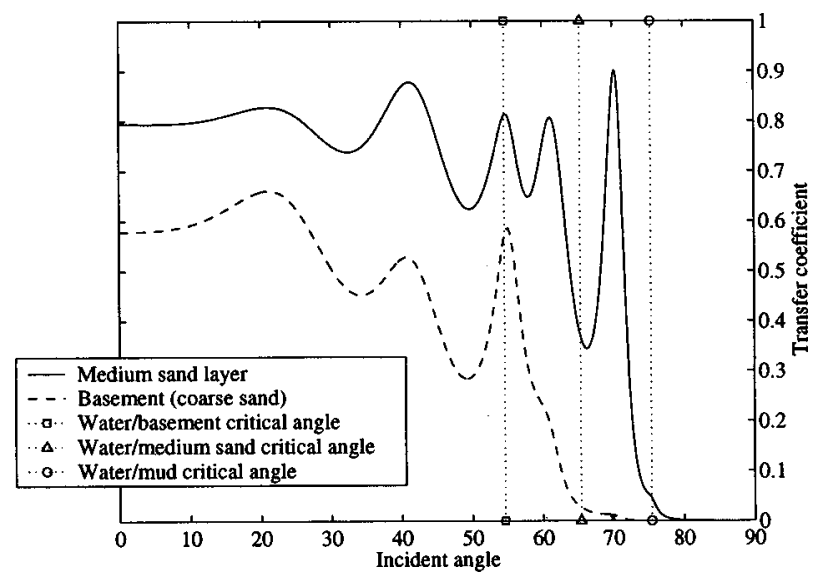

FIG. 10. Transfer coefficients for Table II configuration, computed from sand layer to water (solid curve) and from basement to water (dashed curve).

All the above was developed for the case of depthinvariant characteristics inside each layer, for which the propagating waves are plane, thus making the reflection and transmission coefficients straightforward to compute. Note that the same method of transfer coefficient computation may be readily adapted to the case of layers featuring depthvarying sound speed, density, and attenuation. This was evoked in Refs. 18 and 19 and will be presented with more details in a future paper.

\section{NUMERICAL EXAMPLES}

\section{A. Practical application}

The total EIBS of a geoacoustic configuration may be readily computed from the various elements presented in the previous sections. Several steps are necessary. After the acoustical parameters of each layer are defined (see an example in Table II), the individual BCS (related to their nature and to the geological context) are computed at the various interfaces by using a backscattering model such as Eqs. (3)(5). Then these individual BCSs are modified according to the acoustical parameters of the sediment stack in order to define the local BCS, as described in Sec. III A and using Eqs. (6) and (7). The anamorphosis effect due to refraction is accounted for by applying Eq. (8). Next, the transfer coefficients from the various layers to the upper interface $C_{p l}\left(\theta_{0}\right)$ are computed using Eqs. (26)-(29). Finally, the total equivalent BCS is obtained by summing all the layer contributions using Eq. (1).

\section{B. Two-layer model}

The first example is the geoacoustic configuration presented in Table II. The results are in Figs. 11 and 12.

The total backscattering strength follows the modified basement backscattering strength on a wide angular range. The strong oscillation at 55 degrees is due to the transfer coefficient $C_{p 4}$ of the basement near the water/basement critical angle (see Fig. 10). Beyond this critical angle, the total backscattering strength decreases rapidly and finally follows the mud layer backscattering strength.

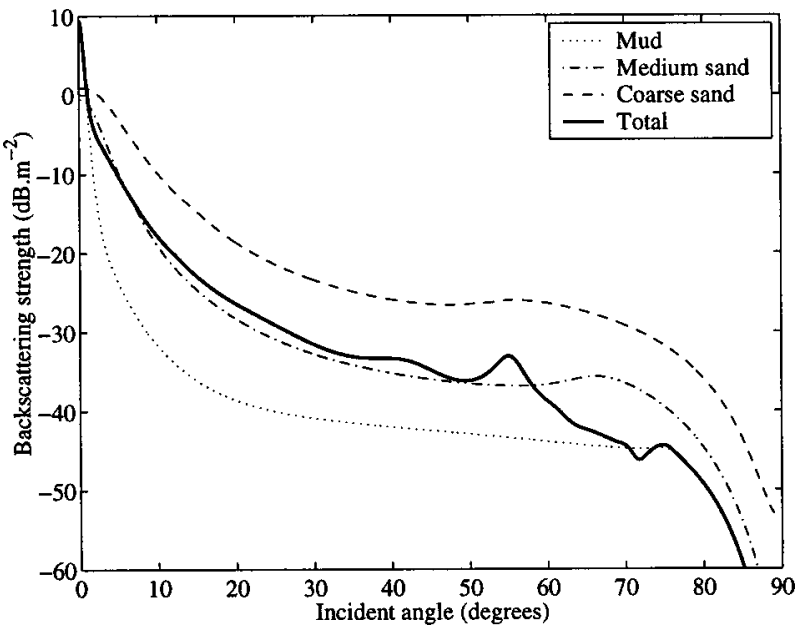

FIG. 11. Individual backscattering strength (dotted curves) with reference to water, and EIBS (solid curve) for the geoacoustic configuration presented in Table II.

\section{Comparison with Ivakin's model}

It is interesting to compare, both formally and in their results, our EIBS model and the "unified approach" proposed by Ivakin. ${ }^{9}$ While his is theoretically far more general than ours, a ground of comparison may be found in the limit case presented by Ivakin as the first-order approximation of his model. In this case corresponding to single-scattering, the two approaches provide nearly identical expressions [see his Eqs. (64) and (65) in Ref. 9].

The two numerical models were compared in various test cases. ${ }^{28}$ We present here a computation of roughness backscattering by a two-layer seafloor (clay and silt layers over a sandy basement) with parameters provided in Table III.

The computation of this seafloor backscattering strength through Ivakin's first-order model is presented in Fig. 13, and computation with the EIBS model is presented in Fig. 14.

The two models are in very good agreement, especially for the location and the amplitudes of the oscillations. There

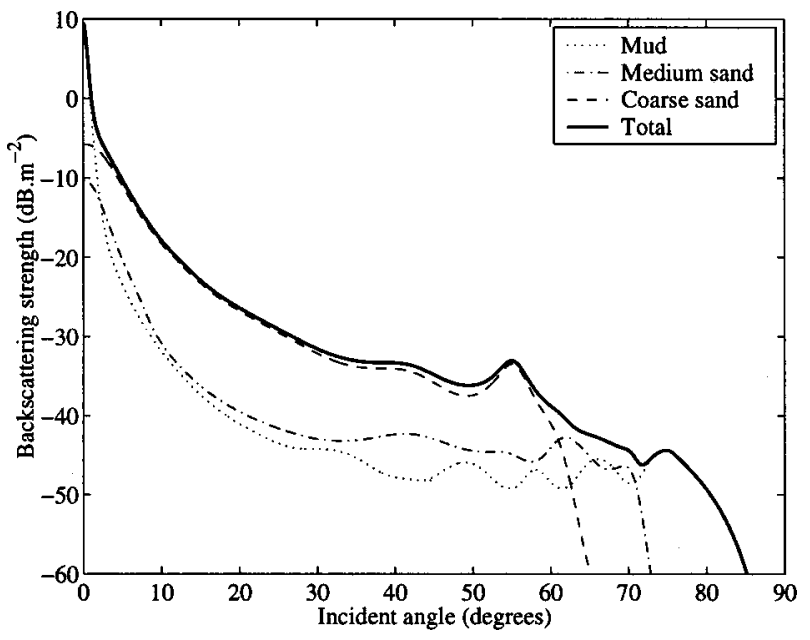

FIG. 12. Local backscattering strength (dotted curves) weighted by their transfer coefficient and EIBS (solid curve) for the geoacoustic configuration presented in Table II. 
TABLE III. Geoacoustic parameters of the second numerical example (comparison with Ivakin's model).

\begin{tabular}{lcccc}
\hline \hline \multicolumn{1}{c}{ Layers: } & Water & Clay & Silt & Sand \\
\hline$c\left(\mathrm{~m} \cdot \mathrm{s}^{-1}\right)$ & 1500 & 1580 & 1650 & 1750 \\
$\rho$ & 1 & 1.5 & 1.8 & 2 \\
$\alpha(\mathrm{dB} / \lambda)$ & 0 & 0.3 & 0.5 & 0.6 \\
$\gamma$ & $\ldots$ & 3.25 & 3.25 & 3.25 \\
$\beta_{J}\left(\mathrm{~cm}^{4}\right)$ & $\ldots$ & $4 \times 10^{-4}$ & $10^{-3}$ & $2 \times 10^{-3}$ \\
$d(\mathrm{~cm})$ & $\ldots$ & 50 & 20 & $\cdots$ \\
\hline \hline
\end{tabular}

are small differences in the prediction of the backscattering strength amplitudes from the two buried interfaces, but the two computation results are very close.

\section{DISCUSSION}

The EIBS model proposed in this article offers a wide potential for the interpretation of experimental backscattered data, in particular those obtained with low-frequency multibeam echosounders on soft sedimentary seafloors for which penetration phenomena are notable. Actually its results have already been compared to experimental data obtained with a $13-\mathrm{kHz}$ multibeam echosounder in two configurations, ${ }^{17,19}$ revealing effects associated with sediment layering.

However, one has to be careful in using such a model for experimental data validation, since its input parameters are numerous and its output is very sensitive to small variations of the configuration. Its modular structure makes it possible to account for complex sedimentary structures. On the other hand, increasing the complexity of the description increases the number of input parameters needed, which may make it difficult to provide the model with numerical values, and may make its results risky to interpret. This raises several issues. First, establishing a geoacoustic model for a given real configuration unavoidably implies relying upon available geological data; however, these are seldom usable directly for the acoustical modeling purpose. For instance, data obtained from geological analyses and geotechnical measurements of sample cores are often very detailed, and

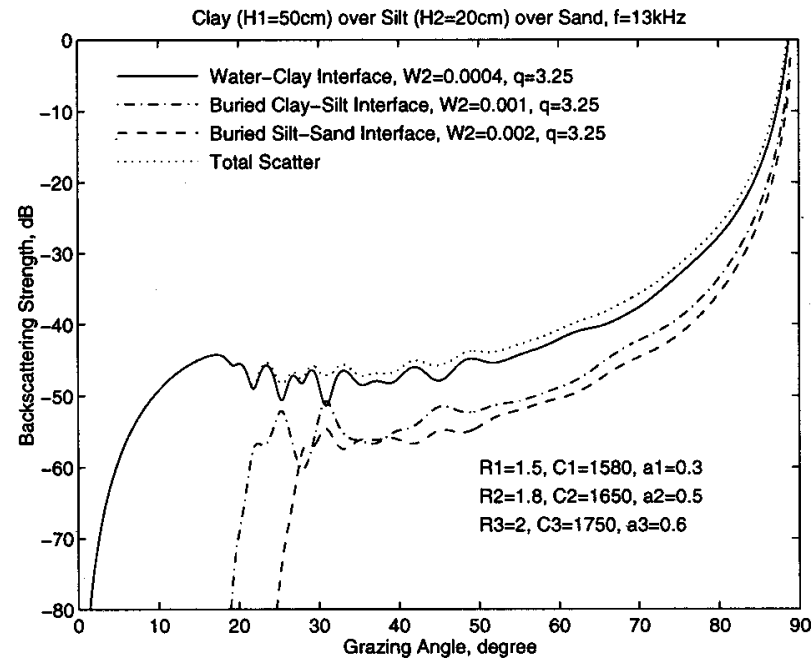

FIG. 13. Local and total backscattering strength for the seafloor model presented in Table III computed with the first order of Ivakin's model (Ref. 9). (This figure is taken from Fig. 1a in Ref. 28 by Ivakin.)

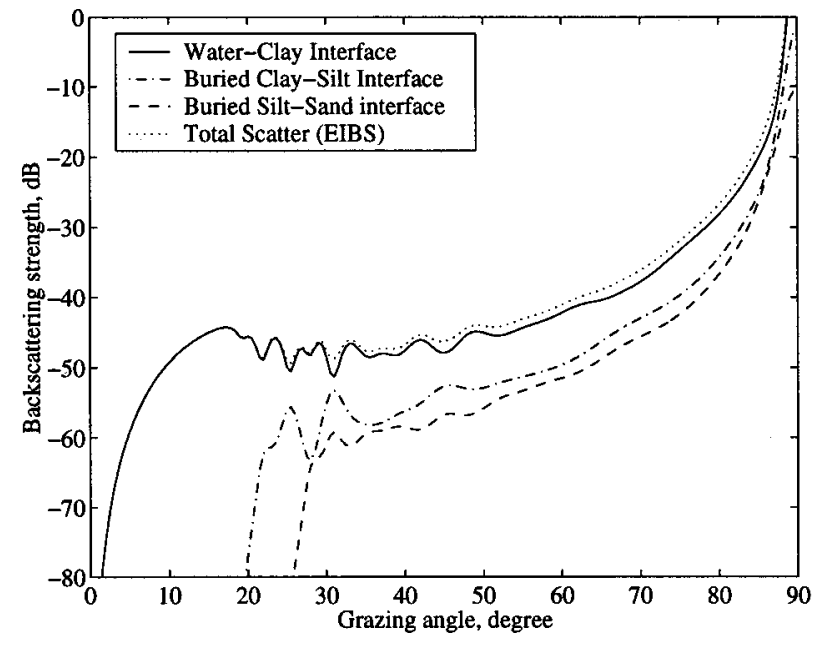

FIG. 14. Local and total backscattering strength for the seafloor model presented in Table III computed with the EIBS model.

they must be simplified to make the acoustical model practicable. Also, the parameters coming from classical geological investigations have to be transformed into usable acoustical parameters, using intermediate models. ${ }^{23}$ Moreover some of the parameters needed in an acoustical model of layered sediments are not accessible to measurement; for example, it is practically impossible today to measure the in situ roughness of buried layers. Finally, because of the high number of input parameters and the limited information usually obtainable from the echosounder experimental data, ambiguities may affect the interpretation: several different layered structures may provide more or less the same response measured as an average intensity level. ${ }^{19}$

The theoretical limitations of the approach should also be kept in mind. On one hand its overall validity depends on the model used for local phenomena, and the transferred backscattering strength cannot be expected to be more accurate than the initial local one. We did not propose anything new in this respect; we just present the local modifications to be applied to a given classical model. Moreover, the EIBS model itself is defined under the limitation of small perturbations and single scattering, and hence its results should be taken cautiously in the regimes of high-level scattering such as the near-specular incidences.

To summarize, the EIBS model presented is basically a formalization of a physically intuitive approach, that makes it possible to describe the backscattering strength associated with buried layers, considered as local phenomena filtered by the overlying sediment structure. Such a physical concept has already been presented and exploited (see, e.g., Refs. 2, 13, and 15), but we believe that the EIBS approach is more general and easier to use because of its detailed description of the local backscattering phenomena modifications, its easy extension to any number of layers, and its potential for processing sedimentary characteristics that continuously vary with depth. On the other hand, it agrees in a satisfactory way with developments obtained from a general theoretical approach of the problem. ${ }^{9}$ The EIBS model is then proposed as a practical compromise between a pragmatic approach of the 
physical problem and a rigorous treatment of the backscattering phenomena.

\section{ACKNOWLEDGMENTS}

The authors wish to thank Anatoliy Ivakin (Andreev Acoustics Institute, Moscow) for making his own results available for publication here, and for many fruitful discussions. We also thank Bruno Lombard and Joel Piraux (LMA-CNRS) for their comments and suggestions about this paper.

\section{APPENDIX: PHASE DIFFERENCE FOR BACKSCATTERED WAVE}

Considering an image source $\mathrm{A}^{*}$ at depth $h^{*}$ (cf. Fig. 7 but the following is valid whatever the image-source order), the phase difference between paths $\mathrm{AS}$ and $\mathrm{A} * \mathrm{~S}$ is developed as

$$
\begin{aligned}
\Delta \varphi= & k_{0} H\left(\frac{1}{\cos \theta_{0,1}}-\frac{1}{\cos \theta_{0}}\right)+k_{1}\left(\frac{h^{*}}{\cos \theta_{1,1}}-\frac{h}{\cos \theta_{1}}\right) \\
= & k_{0} H\left(\frac{1}{\cos \theta_{0,1}}-\frac{1}{\cos \theta_{0}}\right)+k_{1} h^{*}\left(\frac{1}{\cos \theta_{1,1}}-\frac{1}{\cos \theta_{1}}\right) \\
& +k_{1}\left(h^{*}-h\right) \frac{1}{\cos \theta_{1}} .
\end{aligned}
$$

Taking into account that $H \gg h$, variations in $h$ will slightly modify $\theta_{0}$ and $\theta_{1}$. Therefore, the difference $1 / \cos \theta^{*}-1 / \cos \theta$ may be written as the differential $(\partial / \partial \theta)$ $\times(1 / \cos \theta)$. Equation (A1) becomes

$$
\begin{aligned}
\Delta \varphi= & k_{0} H \frac{\sin \theta_{0}}{\cos ^{2} \theta_{0}} \partial \theta_{0}+k_{1} h^{*} \frac{\sin \theta_{1}}{\cos ^{2} \theta_{1}} \partial \theta_{1} \\
& +k_{1}\left(h^{*}-h\right) \frac{1}{\cos \theta_{1}} .
\end{aligned}
$$

Neglecting the second-order terms leads to the following expression for the phase difference:

$$
\Delta \varphi=k_{0} H \frac{\sin \theta_{0}}{\cos ^{2} \theta_{0}} \partial \theta_{0}+k_{1}\left(h^{*}-h\right) \frac{1}{\cos \theta_{1}} .
$$

The relation between variations in $h$ and $\theta$ is obtained from the expression of horizontal range $x$ :

$$
x=H \tan \theta_{0}+h \tan \theta_{1},
$$

the differential of which gives, neglecting the second orderterms,

$$
\partial \theta_{0}=-\tan \theta_{1} \cos ^{2} \theta_{0} \frac{\partial h}{H} .
$$

Using Eq. (A5) in Eq. (A3) leads to

$$
\Delta \varphi=-k_{0} \sin \theta_{0} \tan \theta_{1} \partial h+k_{1} \frac{h^{*}-h}{\cos \theta 1} .
$$

Rewriting this expression with the use of the SnellDescartes relation [Eq. (8)] gives

$$
\begin{aligned}
\Delta \varphi & =-k_{1} \frac{\sin ^{2} \theta_{1}}{\cos \theta_{1}} \partial h+k_{1} \frac{h^{*}-h}{\cos \theta_{1}} \\
& =k_{1} \cos \theta_{1}\left(h^{*}-h\right)=k_{z_{1}}\left(h^{*}-h\right) .
\end{aligned}
$$

Hence, the phase difference between an image-source geometrical path and the direct path is simply approximated by the corresponding plane wave phase difference. Since for image $(m), h^{*}=(2 m+1) h$, it becomes

$$
\Delta \varphi_{m}=2 k_{z_{1}} m h
$$

${ }^{1}$ D. R. Jackson, D. P. Winnebrenner, and A. Ishimaru, “Application of the composite roughness model to high frequency bottom backscattering,' J. Acoust. Soc. Am. 79, 1410-1422 (1986).

${ }^{2}$ J. V. Gardner et al., "Ground-truthing $6.5 \mathrm{kHz}$ side scan sonographs: what are we really imaging?" J. Geophys. Res. 96, 5955-5974 (1991).

${ }^{3}$ M. Stern, A. Bedford, and H. R. Millwater, "Wave reflection from a sediment layer with depth-dependent properties,' J. Acoust. Soc. Am. 77, 1781-1788 (1985).

${ }^{4}$ M. A. Ainslie, "Reflection and transmission coefficients for a layered fluid sediment overlying a uniform solid substrate,', J. Acoust. Soc. Am. 99, 893-902 (1996).

${ }^{5}$ R. Carbo, "Wave reflection from a transitional layer between the seawater and the bottom,' J. Acoust. Soc. Am. 101, 227-232 (1997).

${ }^{6}$ A. N. Ivakin, "Sound scattering by random inhomogeneities of stratified ocean sediments,' Sov. Phys. Acoust. 32, 492-496 (1987).

${ }^{7}$ A. N. Ivakin, "Sound scattering by rough interfaces and volume inhomogeneties of a layered seabed,' J. Acoust. Soc. Am. 95, 2884-2885(A) (1994).

${ }^{8}$ A. N. Ivakin, "A unified approach to volume and roughness scattering," J. Acoust. Soc. Am. 98, 2988(A) (1994).

${ }^{9}$ A. N. Ivakin, "A unified approach to volume and roughness scattering," J. Acoust. Soc. Am. 103, 827-837 (1998).

${ }^{10}$ D. Tang, "A note on scattering by a stack of rough interfaces," J. Acoust. Soc. Am. 99, 1414-1418 (1996).

${ }^{11} \mathrm{~S}$. McDaniel, "Effect of surficial sediment layering on high-frequency seafloor reverberation,' J. Acoust. Soc. Am. 91, 1353-1356 (1992).

${ }^{12}$ H. H. Essen, "Scattering from a rough sedimental seafloor containing shear and layering,' J. Acoust. Soc. Am. 95, 1299-1310 (1994).

${ }^{13}$ Q. J. Huggett et al., "Interference fringes on Gloria side-scan sonar images from the Bering Sea and their implications,', Mar. Geophys. Res. 14, 47-63 (1992).

${ }^{14}$ N. C. Mitchell, "A model for attenuation of backscatter due to sediment accumulations and its application to determine sediment thickness with GLORIA sidescan sonar,' J. Geophys. Res. B 98, 22477-22493 (1993).

${ }^{15}$ A. P. Lyons, A. L. Anderson, and F. S. Dwan, "Acoustic scattering from the seafloor: Modeling and data comparison," J. Acoust. Soc. Am. 95, 2441-2451 (1994).

${ }^{16}$ L. Guillon and X. Lurton, "Rétrodiffusion de signaux de sondeurs grands fonds: influence du volume sédimentaire," in Actes du 4ème Congrès Français d'Acoustique, edited by G. Canévet, G. Mangiante, and S. Meunier (Teknea, Toulouse, France, 1997), pp. 1121-1124 (in French).

${ }^{17}$ L. Guillon and X. Lurton, "Backscattering by layered media: Modeling and comparison with data,' J. Acoust. Soc. Am. 103, 2900(A) (1998).

${ }^{18}$ L. Guillon, 'Contribution à l'interprétation géoacoustique de la rétrodiffusion des fonds marins," Ph.D. thesis (text in French), Université du Maine, Le Mans, France, 1999.

${ }^{19} \mathrm{X}$. Lurton and L. Guillon, "Seafloor characterization using a lowfrequency multi-beam echo-sounder: Ambiguities in backscattering strength interpretation,' J. Acoust. Soc. Am. 105, 1206(A) (1999).

${ }^{20}$ L. M. Brekhovskikh and O. A. Godin, Acoustics of Layered Media. I: Plane and Quasi-Plane Waves (Springer-Verlag, Berlin, 1990).

${ }^{21}$ P. C. Hines, "Theoretical model of acoustic backscatter from a smooth seabed,' J. Acoust. Soc. Am. 88, 324-334 (1990).

${ }^{22} \mathrm{~T}$. Yamamoto, "Acoustic scattering in the ocean from velocity and den- 
sity fluctuations in the sediments,', J. Acoust. Soc. Am. 99, 866-879 (1996).

${ }^{23}$ E. L. Hamilton, "Geoacoustic modeling of the sea floor,' J. Acoust. Soc. Am. 68, 1313-1340 (1980).

${ }^{24}$ P. D. Mourad and D. R. Jackson, "High-frequency sonar equation models for bottom backscatter and forward loss," in Proceedings of Oceans' 89 (IEEE, New York, 1989), pp. 1168-1175.

${ }^{25}$ D. R. Jackson and K. B. Briggs, "High-frequency bottom backscattering: Roughness versus sediment volume scattering,' J. Acoust. Soc. Am. 92, 962-977 (1992).
${ }^{26}$ J. E. Moe and D. R. Jackson, "First-order perturbation solution for rough surface scattering cross section including the effects of gradients," J. Acoust. Soc. Am. 96, 1748-1754 (1994).

${ }^{27}$ L. M. Brekhovskikh and Yu. P. Lysanov, Fundamentals of Ocean Acoustics, 2nd ed. (Springer-Verlag, Berlin, 1991), pp. 210-212.

${ }^{28}$ A. Ivakin, "Modelling of Medium Frequency Acoustic Scattering from Marine Sediments with Buried Interfaces,' Contract Ifremer No. 98 2311227DITI/SM/ASM, Report of the Andreyev Acoustics Institute (1999). 